\title{
Wide-spectrum biomimetic antimicrobial systems
}

\author{
Heidi A. Wright and Byron F. Brehm-Stecher* \\ Rapid Microbial Detection and Control Laboratory, Department of Food Science and Human Nutrition, \\ lowa State University, Ames, IA 50011, USA \\ *Corresponding author's e-mail address: byron@iastate.edu
}

Published online: 29 December 2016 (version 1)

Cite as: Wright, H.A., Brehm-Stecher, B.F. (2017). Wide-Spectrum biomimetic antimicrobial systems. ScienceOpen Research, 12, 2016 (DOI: 10.14293/S2199-1006.1.SOR-LIFE.CMGPSN.v1)

Reviewing status: Please note that this article is under continuous review. For the current reviewing status and the latest referee's comments please click here or scan the QR code at the end of this article.

Primary discipline: Microbiology \& Virology

Associated disciplines: General Agriculture

Keywords: Biomimetics, Antimicrobial Systems, Flavorant and Aroma Compounds, Sesquiterpenoids

\begin{abstract}
Antimicrobial peptides (AMPs) are effective components of the host immune response and are widely distributed throughout nature. Recently, nontoxic antimicrobial polymers that mimic the structures of naturally occurring AMPs have been designed and are under development commercially as novel therapeutics. These compounds have several potential advantages over natural AMPs, including greater stability and reduced immunogenicity compared to natural peptides, relatively simple and scalable syntheses and the ability to tailor or "fine tune" their activities through combinatorial approaches. In previous work, we demonstrated the utility of certain generally regarded as safe (GRAS) flavorant and aroma compounds as enhancers of uptake and activity of clinically important antibiotics (Brehm-Stecher \& Johnson, 2003). Here, we have extended this approach to include enhancement of biomimetic antimicrobial polymers. Three low molecular weight $(<1000 \mathrm{D})$, broad-spectrum arylamide polymers (PolyMedix, Inc., Radnor, PA) were examined for their antimicrobial activities against gram-negative bacteria, gram-positive bacteria, yeast and filamentous fungi, both alone and when co-administered with sesquiterpenoid enhancers. Assay formats included disk diffusion, automated turbidimetry, time course (kinetic) plating of antimicrobial-treated cell suspensions, outer membrane assays with 1-N-phenylnaphthylamine (NPN) and transmission electron microscopy (TEM). Although results differed according to the polymer and test organism used, treatments containing sesquiterpenoids were marked by either increased ZOIs, decreased MICs or more rapid inactivation when compared with polymer-only treatments. Antimicrobial activity, expressed as decimal reduction times (D-value), showed that after $5 \mathrm{~min}$, the combination of sesquiterpenoid and polymer was significantly different from the controls $(p<0.05)$ with a D-value of 3.92 min when incubated with Escherichia coli ATCC 25922. Collectively, our results indicate that the combination of sesquiterpenoid-enhancing agents with biomimetic antimicrobial polymers shows promise for the development of new, faster-acting and more broadly effective antimicrobial therapies.
\end{abstract}

\section{INTRODUCTION}

The recent discovery of the widespread distribution of antimicrobial peptides (AMPs) in multicellular organisms and their essential role in host defense mechanisms has uncovered novel possibilities for therapeutics. Despite the sequence-based diversity of these peptides among organisms, recent work has revealed surprising similarities between conserved structures and antimicrobial function. Many of the peptides yielding substantial antimicrobial activity share similar "facially amphiphilic" structures (Hancock \& Rozek, 2002; Yeaman \& Yount, 2003).

Antimicrobial activity depends largely upon the interaction with the negatively charged bacterial surface, which is the initial target for peptide contact, leading to an induction of amphiphilic structures formation. In gram-negative bacteria, the peptides first interact with the outer membranes that are rich in lipopolysaccharides (LPS), whereas in gram-positive bacteria they transverse the negatively charged peptidoglycan layer rich in teichoic and teichuronic acids (Shai, 2002; Yeaman \& Yount, 2003; Zasloff, 2002). The outer membrane present in gram-negative bacteria results in an additional permeability barrier that may impart increased resistance to certain antimicrobials. The basis for the integrity of the outer membrane is a result of the LPS surface which can potentially bind compounds and decrease their permeability into the interior of the cell (Vaara, 1992). This decreased entrance into the cell has been exploited by knowledge as to the action of AMPs. These unifying structure-function relationships among AMPs have spurred the development of synthetic mimics of these natural peptides in polymeric and small molecule forms. Recently, de novo design of facially amphiphilic biomimetic arylamide polymers has been created that shows antimicrobial activity against a range of bacteria based on hydrophobicity and charge of the side chains (Tew et al., 2002). Polymethacrylate derivatives have also been designed and evaluated for key biological properties that impact antimicrobial activity therefore allowing for the design of synthetic 
polymers that are inexpensive to prepare (Kuroda \& DeGrado, 2005). This highlights one of the advantages of biomimetic polymers over natural peptides, including their lower cost of production and the ability to scale synthesis to industrially relevant production outputs. The insights gained from studies on AMPs may provide new strategies and templates for the development and design of novel biomimetics, and they may be used in therapeutic, pharmaceutical, and food applications.

As these mimics target the cell membrane (a highly conserved target), widespread microbial resistance is not expected to develop toward these compounds. This feature is important due to the increasing instance of bacterial resistance to conventional antibiotics which typically function by inhibiting key bacterial enzymes. Bacteria can evade these mechanisms and develop resistance; however, targeting of central structural features by AMPs makes them more potent due to the decreased potential for developed resistance (Lockwood \& Mayo, 2003). In addition, the ability of these mimics to exploit a separate pathway for antimicrobial or antibiotic uptake allows for their effectiveness against a broad spectrum of microorganisms, including gram-negative bacteria and fungi. Traditional antibiotics typically have a limited spectrum of activity that decreases their potential range for applications, especially relating to the additional selectivity barrier inherent in gram-negative bacteria (Hancock, 1997, 2001; Hancock \& Rozek, 2002; Zasloff, 2002). By targeting the membrane instead of specific metabolic pathways or enzymes, resistance issues may be avoided, and the potential for an increased antimicrobial spectrum of activity may be created.

Although these novel biomimetics have desirable properties, their antimicrobial activity may still be lessened against gramnegative bacteria due to the extra outer membrane. However, certain compounds which alone may be weakly antimicrobial have been shown to sensitize gram-negative bacteria to antimicrobial agents by disrupting the outer membrane (Vaara, 1992). Generally regarded as safe (GRAS) sesquiterpenoids have previously been shown to be effective antimicrobial-enhancing agents, especially for membrane-targeted antimicrobials (Brehm-Stecher \& Johnson, 2003). Because the biomimetic polymers we are working with act primarily at microbial membranes, we sought here to combine them with sesquiterpenoid enhancers in order to create new, high potency antimicrobial formulations.

\section{MATERIALS AND METHODS Culture of microorganisms}

Escherichia coli ATCC 25922 and Staphylococcus aureus ATCC 29523 were cultured in cation-adjusted Mueller Hinton broth (MH) (Becton Dickinson and Company, Franklin Lakes, N.J.) as recommended by CLSI for bacteria that grow aerobically (M07-A9; CLSI 2012) Trichophyton mentagrophytes ATCC (9533) was prepared according to CLSI recommendations for filamentous fungi (M38-A2, CLSI 2008a) with the following modifications. Fungi were grown on CAMHA at $35^{\circ} \mathrm{C}$ for 7 days to produce conidia (Santos et al., 2006). The conidia were recovered by wetting a loop with Tween 20 and transferring the loopful of conidia into $5 \mathrm{ml}$ of sterile saline $(0.9 \%)$. After the mixture of conidia and hyphal fragments was allowed to settle for 15-20 min at room temperature, the upper suspension was transferred to a sterile tube (Espinel-Ingroff \& Canton, 2007). Candida albicans ATCC (90028) was also cultured in RPMI 1640 standard broth according to CLSI guidelines (M27-A3, CLSI 2008b) overnight at $35^{\circ} \mathrm{C}$.

\section{Antimicrobial polymers and sesquiterpenoids}

Biomimetic antimicrobial polymers were sourced from PolyMedix, Inc. (Radnor, PA). The polymers used were polymethacrylates arylamide oligomers (M.W. <2.000) and included butyl methacrylate (PMX 50001), butyl methacrylate with a different percent composition of monomers (PMX 50003), and ethyl methacrylate (PMX 50004). Stock solutions of the polymers as $10 \mathrm{mg} / \mathrm{mL}$ were prepared in 1:1 methanol and water and stored at $-20^{\circ} \mathrm{C}$ under dry conditions. Sesquiterpenoids, such as bisabolol, farnesol, and nerolidol, were sourced from Sigma-Aldrich (St. Louis, MO) with stock solutions prepared to $10,000 \mu \mathrm{M}$ in propylene glycol (DOW Chemical Company, Midland, MI).

\section{Agar diffusion}

The endpoint disk diffusion assay was used as a measure to determine the ability of sesquiterpenoids to sensitize culture organisms to antimicrobial polymers. Methods were followed as recommended by CLSI (M2-A9, M07-A9, CLSI 2006, 2012) with modifications described briefly. Cells were grown overnight (24 h) at $30^{\circ} \mathrm{C}$ or $35^{\circ} \mathrm{C}$ in CAMHB with dilution in neutral phosphate buffer. An aliquot was combined with a $0.7 \%$ Mueller Hinton agar overlay tempered to $50^{\circ} \mathrm{C}$ to achieve a final inoculum size of $10^{6} \mathrm{cfu} / \mathrm{mL}$. Sesquiterpenoid stock solutions in propylene glycol were added in appropriate combinations to the celloverlay mixture to a final concentration of $200 \mu \mathrm{M}$. The mixture was immediately poured over hardened CAMHA in $60 \mathrm{~mm}$ diameter petri dishes. Sterile paper disks (BBL, Becton Dickson and Company, Franklin Lakes, NJ) were applied an aliquot of the antimicrobial, placed on the hardened agar overlays, and incubated at $30^{\circ} \mathrm{C}$ for $24 \mathrm{~h}$. Diameters of zones (in mm) taken as a measure of antimicrobial activity were measured from the bottom of each plate following incubation.

\section{MIC determination}

The minimum inhibitory concentrations (MICs) were determined using a Bioscreen C (Labsystems, Helsinki, Finland). The Bioscreen Microbial Growth Analyzer is a self-contained microbial incubation and analysis unit that can evaluate changes in turbidity of up to 200 samples $(2 \times 100$ multichannel plates) over time, ranging from several hours to several days. Antimicrobial dilutions were carried out as described previously (Lambert et al., 1998) with modifications 
briefly explained for the enhancement with sesquiterpenoids. In these assays, stock solutions were made into CAMHB with final antimicrobial dilutions ranging from $125 \mu \mathrm{g} / \mathrm{mL}$ to $0.5 \mu \mathrm{g} / \mathrm{mL}$. In combination assays with sesquiterpenoids, stock solutions were again prepared in CAMHB with a final sesquiterpenoid concentration of $100 \mu \mathrm{M}$. A set of control wells was prepared for each experiment with CAMHB added to the control wells to bring the final volume in all wells to the same volume. The plates were incubated in the Bioscreen for $24 \mathrm{~h}$ at $30^{\circ} \mathrm{C}$ for bacteria and $35^{\circ} \mathrm{C}$ for yeasts and molds with optical density measurements $(600 \mathrm{~nm})$ taken every $15 \mathrm{~min}$ with shaking $10 \mathrm{sec}$ prior. MICs were defined as the lowest concentration of antimicrobial that completely inhibited growth (OD increase $\leq$ 0.05) (Branen \& Davidson, 2004).

\section{Antimicrobial kinetics}

The kinetics of antimicrobial killing was preformed to measure the enhanced susceptibility of target culture cells to antimicrobial agents in the presence of the sesquiterpenoids and to quantify the speed and extent to which the inactivation occurred. Polymers were added to overnight culture cells at $31.25 \mu \mathrm{g} / \mathrm{mL}$ with sesquiterpenoids being added to the system at $200 \mu \mathrm{M}$. Aliquots were removed at certain time intervals $(0,5,10,15,30 \mathrm{~min})$ for $E$. coli and $(0,30,60 \mathrm{~min})$ for $C$. albicans with immediate dilution D/E (Dey/Engley) neutralizing broth (Becton Dickinson and Company, Franklin Lakes, N.J.) to prevent residual effects of the antimicrobials. A $0.01 \mathrm{ml}$ aliquot of each dilution was track plated on D/E neutralizing agar according to the guidelines of Jett et al. (1997) and incubated for $24 \mathrm{~h}$ at $30^{\circ} \mathrm{C}$ or $35^{\circ} \mathrm{C}$. Following incubation, survival rates were based on survival comparisons to the untreated bacterial control. D-values for treatments over time were calculated from the survivor curves by plotting the surviving population $(\log 10)$ against antimicrobial treatments at each time point. The equation of the best fit line was obtained through linear regression analysis. The D-values were determined as the interval of time required for the survivor curve to provide a one decimal logarithm $\left(1-\log _{10}\right)$ or $90 \%$ reduction in the initial viable bacterial population and are graphically equivalent to the negative inverse slope of the regression line (Jay, 2005). Both surviving population counts and D-values were analyzed using the general linear model procedure of the SAS statistical software (SAS Institute Inc., Cary, NC). Comparisons between treatments were made by Tukey's student range test $(p<0.05)$.

\section{D/E neutralization test}

In order to verify that $\mathrm{D} / \mathrm{E}$ agar was suitable for neutralizing the activities of the polymers examined here, a D/E agar neutralization test was performed according to the Difco and BBL Manual of Microbiological Culture Media with modifications briefly described. Test organisms were grown overnight and diluted in neutral phosphate buffer. An aliquot of the bacterial culture was combined with a $0.7 \%$ agar overlay and immediately poured over hardened D/E neutralizing agar. The agar plates were swirled to ensure even distribution of the test organism throughout the overlay. Sterile paper disks (BBL, Becton Dickson and Company, Franklin Lakes, NJ) were applied with the antimicrobial, placed on hardened agar overlays, and incubated overnight. Zones of inhibition were compared to zones seen on simultaneous tests of antimicrobial activity on CAMHA. D/E neutralizing agar exhibited no zones of inhibition when compared to the activity on CAMHA, suggesting that the antimicrobial activity was neutralized and residual activity of the polymers upon plating was minimized.

\section{Outer membrane permeabilization}

Permeabilization of the outer membrane was determined by a 1-N-phenylnaphthylamine (NPN) assay. E. coli cells were centrifuged at $2000 \times g$ for $5 \mathrm{~min}$ and resuspended in neutral phosphate buffer at $10^{7} \mathrm{cfu} / \mathrm{mL}$. Cells were placed in a $1-\mathrm{cm}$ cuvette, and changes in fluorescence were monitored with a Cary Eclipse fluorometer (Varian) at an excitation wavelength of $350 \mathrm{~nm}$, an emission wavelength of $420 \mathrm{~nm}$, and slit widths of $5 \mathrm{~nm}$. The background fluorescence was monitored for $10 \mathrm{sec}$ before addition of NPN at a final concentration of 10 $\mu$ M. PMX $50003(31.25 \mu \mathrm{g} / \mathrm{mL})$ and nerolidol $(100 \mu \mathrm{M})$ were tested alone and in combination for their ability to permeabilize cells to NPN.

\section{Transmission electron microscopy}

For transmission electron microscopy (TEM)-based investigations of polymer effects on cellular ultrastructure, PMX 50003 was added to an overnight culture of Escherichia coli ATCC 25922 in CAMBH $\left(\sim 10^{9} \mathrm{CFU} / \mathrm{ml}\right)$ at either $1 \times$ or $2 \times$ MIC (31.25 $\mu \mathrm{g} \mathrm{ml}$ or $62.5 \mu \mathrm{g} \mathrm{ml}$, respectively), with or without nerolidol added at $100 \mu \mathrm{M}$. These treatments (no-antimicrobial control, nerolidol control, 50003 control, and nerolidol + 50003) were mixed thoroughly and aliquots were removed at specific time intervals $(0,5$, and $15 \mathrm{~min})$. Upon removal, aliquots were fixed with the addition of EM-grade glutaraldehyde (2.5\% final concentration) and shipped to the University of Iowa's Central Microscopy Research Facility (Iowa City, IA) for additional preparation prior to TEM.

\section{RESULTS \\ Agar diffusion}

Bisabolol, farnesol, and nerolidol enhanced the activity of all three polymers tested against E. coli, S. aureus, and C. albicans, with farnesol being the most effective enhancer. Nerolidol and bisabolol showed similar enhancing potential at the levels tested for all three organisms. When the sesquiterpenoids were combined with the polymers, the lawns became less dense compared to the controls for $S$. aureus (data not shown). The addition of the sesquiterpenoids increased the 
zones of inhibition along with an overall general clearing of the bacterial lawn. This could be evidence of slight intrinsic antimicrobial activity that has been shown to be present in the sesquiterpenes farnesol and nerolidol. Farnesol and nerolidol have both previously been shown to reduce the initial $S$. aureus viable cell load on a dose-dependent basis with the proposed mechanisms being cell membrane damage (Inoue et al., 2004).

\section{Minimum inhibitory concentrations}

MICs of the biomimetic antimicrobial polymers alone and in combination with sesquiterpenoids are presented in Table 1. Results of the negative controls containing either 1:1 methanol and water or propylene glycol at the highest final concentration in the system indicated an absence of inhibition for all organisms tested (data not shown). All of the

Table 1. Minimum inhibitory concentrations of PMX polymers and enhancement by sesquiterpenoids against select microorganisms.

\begin{tabular}{|c|c|c|c|c|}
\hline \multirow[b]{3}{*}{ Polymer } & \multicolumn{4}{|c|}{$\begin{array}{c}\text { E. coli ATCC } 25922 \\
\text { Minimum inhibitory concentration }(\mu \mathrm{g} / \mathrm{mL})\end{array}$} \\
\hline & \multicolumn{4}{|c|}{ Sesquiterpenoid $(100 \mu \mathrm{M})$} \\
\hline & Control & + Bisabolol & + Farnesol & + Nerolidol \\
\hline 50001 & 31.25 & 15.63 & 7.81 & 15.63 \\
\hline 50003 & 31.25 & 3.91 & 1.95 & 3.91 \\
\hline \multirow[t]{3}{*}{50004} & 62.5 & 3.91 & 3.91 & 3.91 \\
\hline & \multicolumn{4}{|c|}{$\begin{array}{c}\text { S. aureus ATCC } 25923 \\
\text { Minimum inhibitory concentration }(\mu \mathrm{g} / \mathrm{mL})\end{array}$} \\
\hline & \multicolumn{4}{|c|}{ Sesquiterpenoid $(100 \mu \mathrm{M})$} \\
\hline Polymer & Control & + Bisabolol & + Farnesol & + Nerolidol \\
\hline 50001 & 31.25 & 15.63 & 3.91 & 15.63 \\
\hline 50003 & 15.63 & 3.91 & 0.98 & 3.91 \\
\hline \multirow[t]{3}{*}{50004} & 31.25 & 3.91 & 0.98 & 3.91 \\
\hline & \multicolumn{4}{|c|}{$\begin{array}{c}\text { C. albicans ATCC } 90028 \\
\text { Minimum inhibitory concentration }(\mu \mathrm{g} / \mathrm{mL})\end{array}$} \\
\hline & \multicolumn{4}{|c|}{ Sesquiterpenoid $(100 \mu \mathrm{M})$} \\
\hline Polymer & Control & + Bisabolol & + Farnesol & + Nerolidol \\
\hline 50001 & 62.5 & 31.25 & 31.25 & 31.25 \\
\hline 50003 & 31.25 & 15.63 & 7.81 & 15.63 \\
\hline \multirow[t]{3}{*}{50004} & 62.5 & 31.25 & 31.25 & 31.25 \\
\hline & \multicolumn{4}{|c|}{$\begin{array}{l}\text { T. mentagrophytes ATCC } 9533 \\
\text { Minimum inhibitory concentration }(\mu \mathrm{g} / \mathrm{mL})\end{array}$} \\
\hline & \multicolumn{4}{|c|}{ Sesquiterpenoid $(100 \mu \mathrm{M})$} \\
\hline Polymer & Control & + Bisabolol & + Farnesol & + Nerolidol \\
\hline 50001 & 62.5 & 15.63 & 15.63 & 31.25 \\
\hline 50003 & 31.25 & 7.81 & 15.63 & 15.63 \\
\hline 50004 & 62.5 & 15.63 & 15.63 & 31.25 \\
\hline
\end{tabular}

polymers exhibited antimicrobial activity against the four organisms tested with MICs ranging from $15.63 \mu \mathrm{g} / \mathrm{mL}$ to $62.5 \mu \mathrm{g} / \mathrm{mL}$. The polymers alone were overall most effective against the gram-positive $S$. aureus with 50003 exhibiting the greatest antimicrobial activity with an MIC of $15.63 \mu \mathrm{g} / \mathrm{mL}$. The enhancement with sesquiterpenoids against $S$. aureus was the greatest with farnesol, exhibiting a fourfold increase in activity, whereas bisabolol and nerolidol each had a threefold increase. PMX polymer 50001 had the same MIC alone as PMX 50004; however, enhancement was not as profound with 50001. All polymers had higher MIC values against the yeast and mold, C. albicans and T. mentagrophytes, with enhancement by the sesquiterpenoids being greater for T. mentagrophytes in all cases except for the combination of 50003 and farnesol with C. albicans. The lower MIC values for 50003 and increased enhancement in the presence of the sesquiterpenoids as compared to 50001 highlight the potential importance of monomer composition due to the only difference between these two butyl methacrylate polymers being their composition of monomers. This could indicate the significance that different compositional arrangements could play in both antimicrobial activity and in the potential increased uptake by cells when combined in formulations.

\section{Antimicrobial kinetics}

Kinetics of inactivation methods resulted in an immediate one $\log$ reduction of E. coli growth when PMX 50003 was combined with $200 \mu \mathrm{M}$ nerolidol. Decimal reduction times were used to indicate the rapid extent of combination on enhancing antimicrobial activity (Table 2).

The combination of PMX 50003 and nerolidol had a D-value of 3.92 min compared to either 50003 (88.39 min) or nerolidol (260.80 $\mathrm{min}$ ) when assayed alone. After $5 \mathrm{~min}$, there was a significant difference in survivor counts between the combination polymer and nerolidol and either of the control polymer or

Table 2. Enhancement of E. coli ATCC 25922 inactivation with PMX polymer $50003(31.25 \mu \mathrm{g} / \mathrm{ml})$ and nerolidol $(200 \mu \mathrm{M})$.

\begin{tabular}{lcccccc}
\hline & \multicolumn{5}{c}{$\begin{array}{c}\text { Survivor counts } \\
\text { per treatment time }\end{array}$} & \\
\cline { 2 - 6 } Time (min) & $\mathbf{0}$ & $\mathbf{5}$ & $\mathbf{1 0}$ & $\mathbf{1 5}$ & $\mathbf{3 0}$ & D-value $^{\mathbf{2}}$ (min) \\
\hline 50003 & $8.75^{\mathrm{ab}}$ & $8.69^{\mathrm{a}}$ & $8.65^{\mathrm{a}}$ & $8.64^{\mathrm{a}}$ & $8.59^{\mathrm{a}}$ & $88.39^{\mathrm{x}}$ \\
Nerolidol & $8.83^{\mathrm{a}}$ & $8.80^{\mathrm{a}}$ & $8.79^{\mathrm{a}}$ & $8.79^{\mathrm{a}}$ & $8.77^{\mathrm{a}}$ & $260.80^{\mathrm{y}}$ \\
$50003+$ & $8.21^{\mathrm{b}}$ & $5.42^{\mathrm{b}}$ & $4.85^{\mathrm{b}}$ & $4.12^{\mathrm{b}}$ & $3.99^{\mathrm{b}}$ & $3.92^{\mathrm{z}}$ \\
$\quad$ Nerolidol & & & & & & \\
\hline
\end{tabular}

${ }^{1}$ Survivor counts were reported as mean values of three replications.

${ }^{2}$ Decimal reduction time (D-value) is the unit time of exposure that will results in a single $\log _{10}$ reduction in E. coli population.

${ }^{a, b}$ Microbial counts at individual time points followed by the same letter/s are not significantly different at $(p>0.05)$.

${ }_{x, y, z} \mathrm{D}$-values on the same row followed by the same letter/s are not significantly different $(p>0.05)$. 
sesquiterpenoid alone. This significant difference remained through the entire 30-min assay with a continued decrease in survivor counts for the combination throughout (Figure 1).

Kinetics of inactivation with $C$. albicans showed similar trends; however, the extent of inactivation was not measured over 60 min due to the slower inactivation of $C$. albicans at subinhibitory concentrations with PMX 50004 (31.25 $\mu \mathrm{g} / \mathrm{mL})$. The combination of 50004 and bisabolol caused a one log reduction in survivor counts of $C$. albicans within $12.65 \mathrm{~min}$ compared to 50004 (47.35 $\mathrm{min}$ ) and bisabolol (122.55 $\mathrm{min}$ ) alone (Table 3).

The combination after 30 min was also significantly different from the individual compounds indicating that the combination had increased activity over each component alone (Figure 2).

\section{Outer membrane permeabilization}

NPN was used as an indicator of PM permeabilization. When embedded in a more hydrophobic environment, the quantum yield of NPN increases, making it an effective reporter of OM
Table 3. Enhancement of C. albicans ATCC 90028 inactivation with PMX polymer $50004(31.25 \mu \mathrm{g} / \mathrm{ml})$ and bisabolol $(200 \mu \mathrm{M})$.

\begin{tabular}{lcccc}
\hline & \multicolumn{3}{c}{$\begin{array}{c}\text { Survivor counts } \\
\text { per treatment time }\end{array}$} & \\
\cline { 2 - 4 } Time (min) & $\mathbf{0}$ & $\mathbf{3 0}$ & $\mathbf{6 0}$ & D-value (min) \\
\hline 50004 & $6.93^{\mathrm{a}}$ & $6.66^{\mathrm{a}}$ & $6.38^{\mathrm{a}}$ & $47.35^{\mathrm{xy}}$ \\
Bisabolol & $7.03^{\mathrm{a}}$ & $6.90^{\mathrm{a}}$ & $6.75^{\mathrm{a}}$ & $122.55^{\mathrm{x}}$ \\
$50004+$ Bisabolol & $6.88^{\mathrm{a}}$ & $5.41^{\mathrm{b}}$ & $5.05^{\mathrm{b}}$ & $12.65^{\mathrm{y}}$ \\
\hline
\end{tabular}

${ }^{1}$ Survivor counts were reported as mean values of three replications. ${ }^{2}$ Decimal reduction time (D-value) is the unit time of exposure that will results in a single $\log _{10}$ reduction in C. albicans population.

${ }^{a, b}$ Microbial counts at individual time points followed by the same letter/s are not significantly different at $(p>0.05)$.

${ }^{x, y} \mathrm{D}$-values on the same row followed by the same letter/s are not significantly different $(p>0.05)$.

damage. Components in the assay were added in a stepwise sequential order to depict the impact of each compound on OM permeabilization. This method showed that NPN alone had some inherent fluorescence. Further addition of

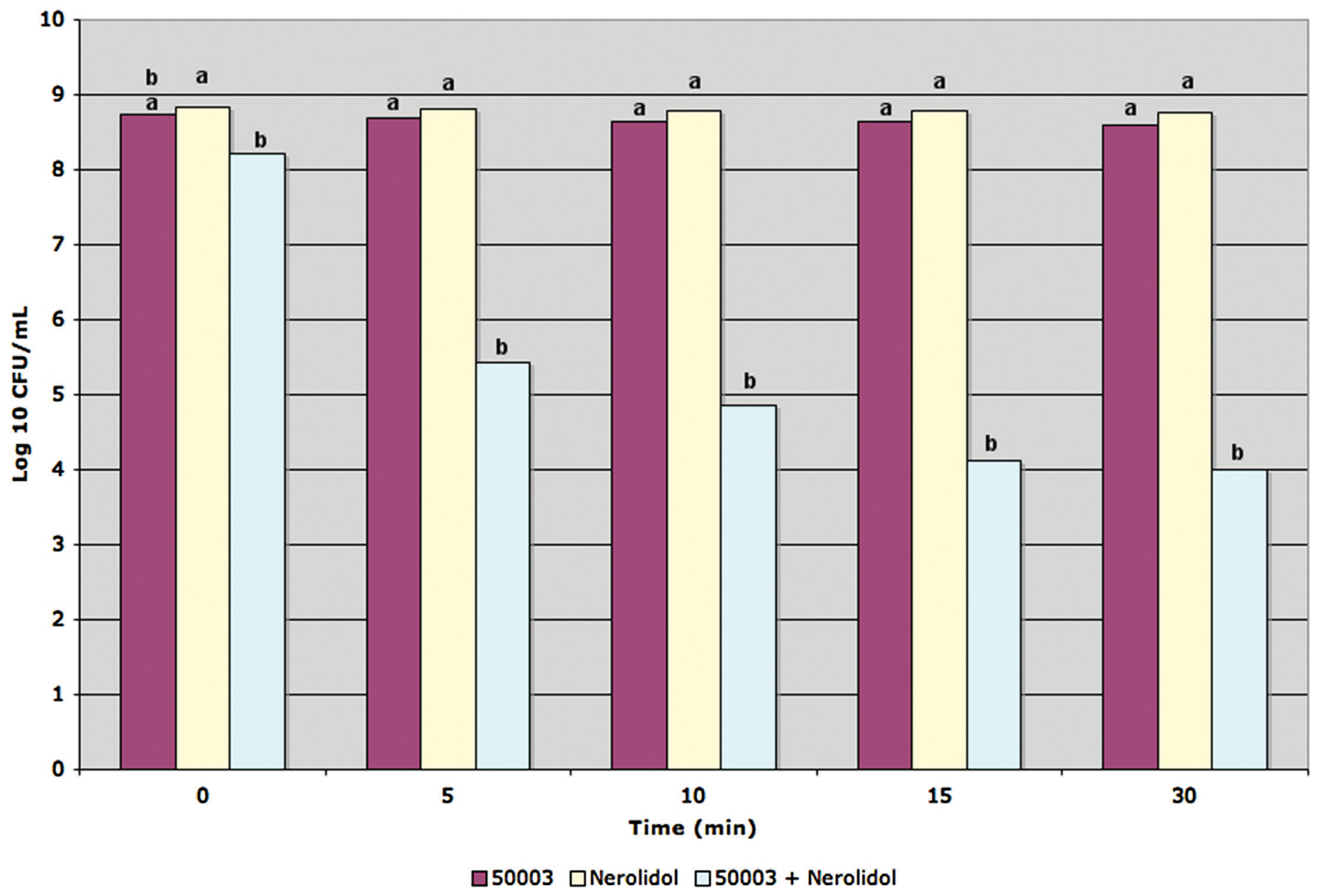

Figure 1. Enhancement of E. coli ATCC 25922 inactivation with PMX polymer 50003. Time course kinetics with 50003 (31.25 $\mu \mathrm{g} / \mathrm{mL}$ ) and enhancement with nerolidol $(200 \mu \mathrm{M})$ sampled at $0,5,10,15$, and $30 \mathrm{~min}$. Values for each time point having the same letter are not significantly different at $p>0.05$. 


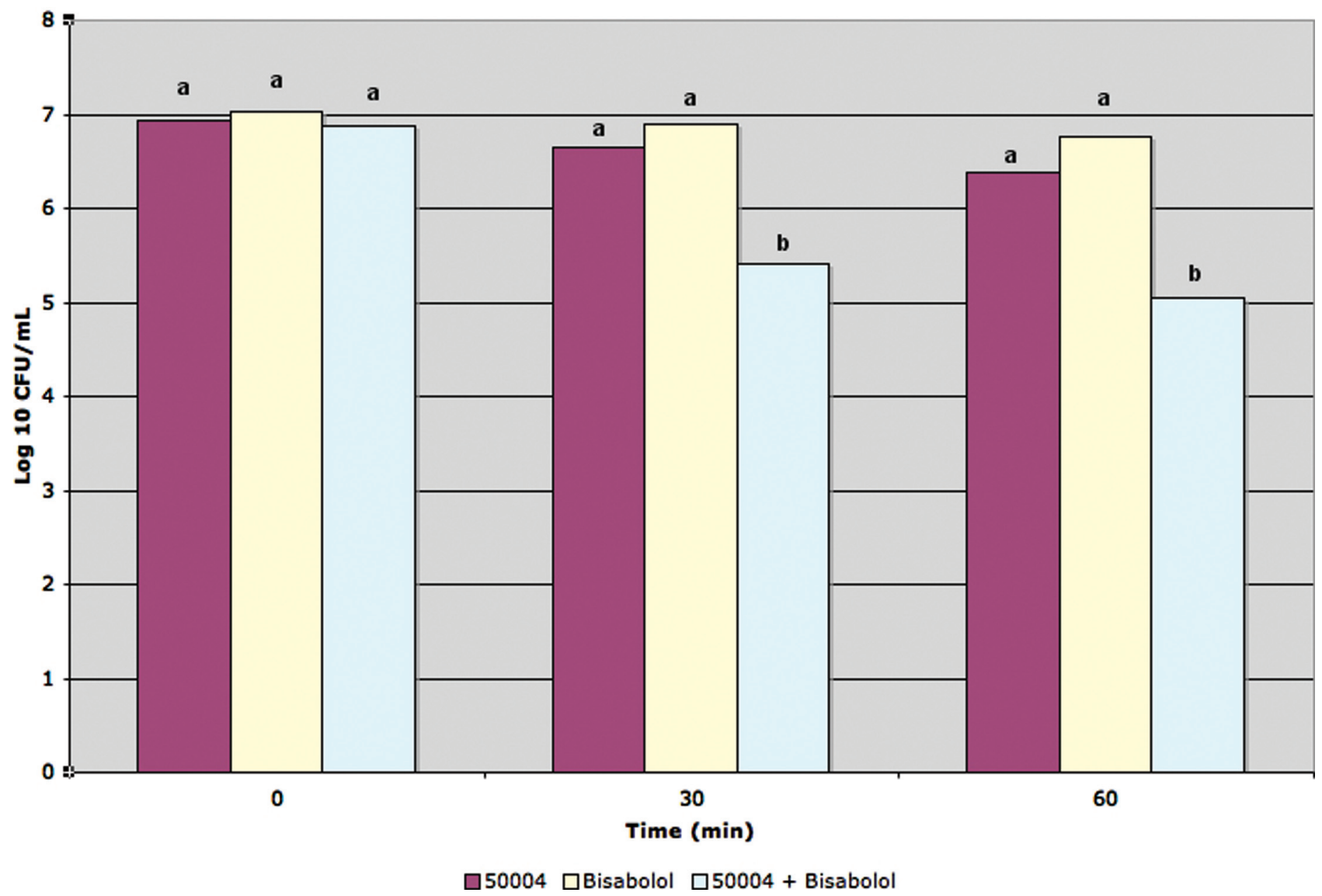

Figure 2. Enhancement of $C$. albicans ATCC 90028 inactivation with PMX polymer 50004. Time course kinetics with 50004 (31.25 $\mu \mathrm{g}$ / $\mathrm{mL}$ ) and enhancement with bisabolol $(200 \mu \mathrm{M})$ sampled at 0,30 , and $60 \mathrm{~min}$. Values for each time point having the same letter are not significantly different at $p>0.05$.

nerolidol only resulted in a slight increase in this baseline fluorescence. When PMX 50003 was added alone with NPN, permeabilization again was only marginally increased. Permeabilization occurred gradually over time and reached a maximal value after $120 \mathrm{sec}$. The addition of NPN, followed by 50003 , and then nerolidol resulted in the same patterns of fluorescence increase for NPN and 50003; subsequent addition of nerolidol resulted in a dramatic increase in fluorescence output (Figure 3).

The rapid cooperative interactions seen in the NPN uptake assay were also observed via TEM, as shown in Figure 4.

\section{DISCUSSION AND CONCLUSIONS Microbial resistance}

Emerging bacterial resistance to antimicrobial drugs represents a significant threat to human health. Market forces affecting industrial R\&D over the past 15 years have led to a diminished pipeline for novel anti-infectives. This is especially true for drugs targeting gram-negative bacteria, where new agents capable of addressing hospital infections are not expected to be available for another 10-15 years. This lack of anti-infectives is particularly worrisome for emerging, drug- resistant human pathogens, against which formerly useful, last line-of-defense antimicrobials are no longer effective.

The traditional "one bug-one drug" concept may no longer be a viable paradigm, given the broad nature of the antimicrobial resistance threat. national institute of allergy and infectious diseases (NIAID's) agenda for future research includes plans to promote discovery and development of natural antimicrobial proteins, including defensins. AMPs, such as defensins, are effective components of the host immune response and are widely distributed throughout nature. Unfortunately, they are expensive to produce in quantity, they are susceptible to enzymatic or chemical degradation, and their structures and activities are not easily manipulated. Nontoxic antimicrobial polymers mimicking the structures of naturally occurring AMPs such as those used here have been designed commercially as novel therapeutics. Advantages of these compounds include relatively simple and scalable syntheses, similar potencies as their natural counterparts, and structural malleability that may allow "fine tuning" of their antimicrobial activities or spectra. The table below provides a comprehensive comparison of the differences between natural AMPs versus biomimetic polymers and the potential advantages of the latter. 


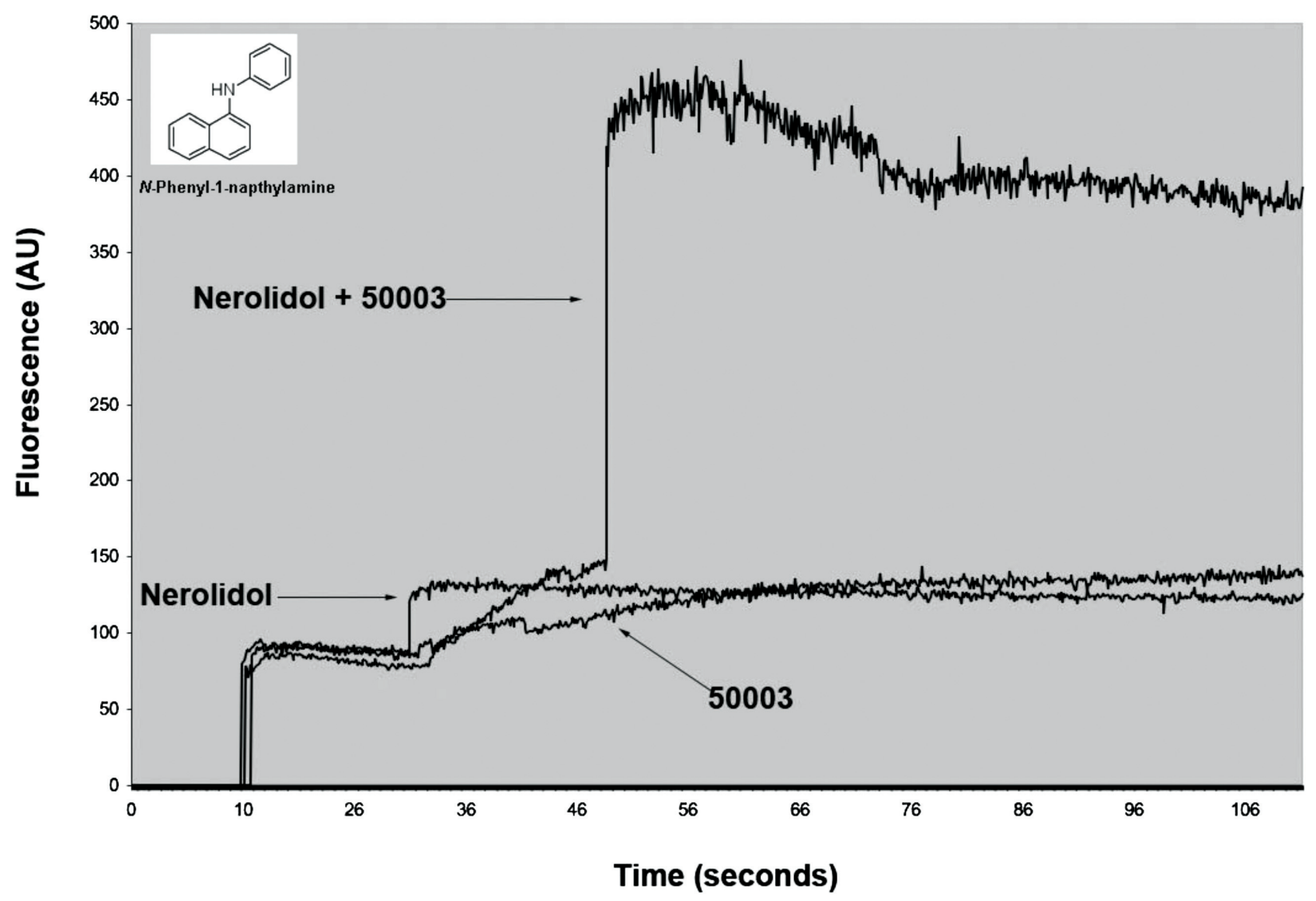

Figure 3. NPN uptake assay with PMX 50003 and nerolidol using E. coli ATCC 25922. Time course of increase in NPN uptake with $50003(31.25 \mu \mathrm{g} / \mathrm{mL})$, nerolidol $(200 \mu \mathrm{M})$ or the combination. NPN at $10 \mu \mathrm{M}$ was added at $10 \mathrm{sec}$ and allowed to stabilize before addition of either nerolidol or $\mathbf{5 0 0 0 3}$ at $\mathbf{3 0} \mathrm{sec}$. When used in combination, the second component was added at $\mathbf{5 0}$ sec. Enhanced uptake of NPN was measured by an increase in fluorescence, the result of partitioning of NPN into the outer membrane.
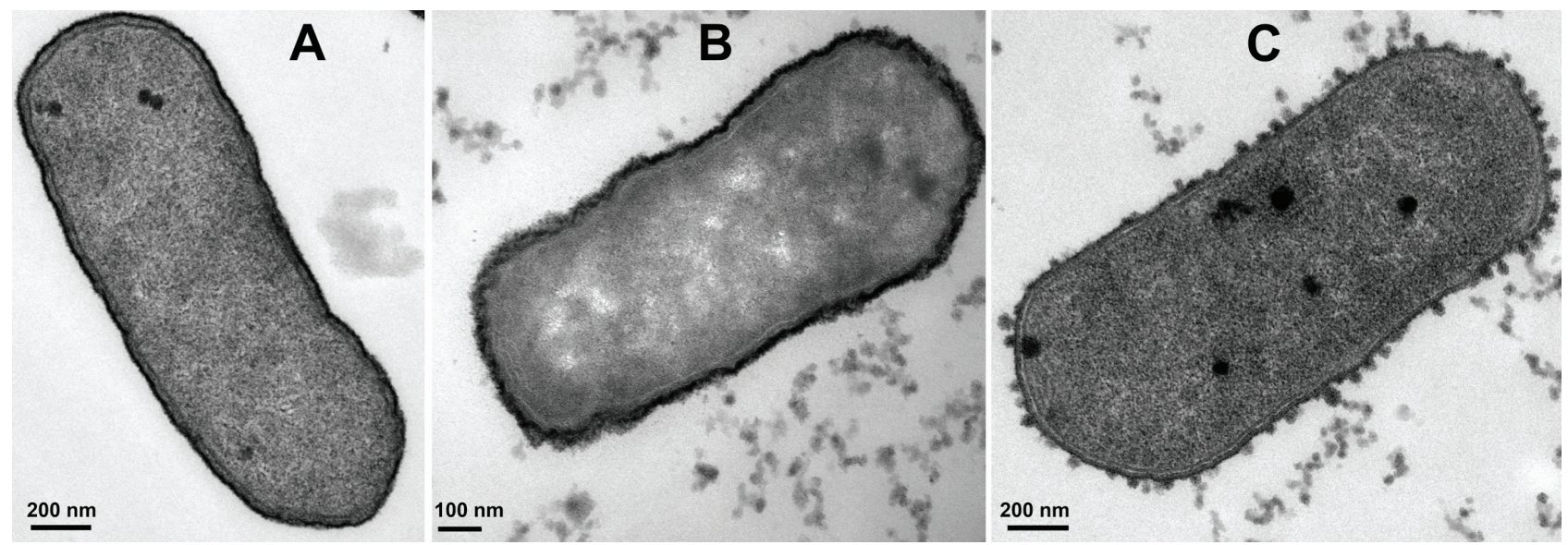

Figure 4. Sesquiterpenoid-mediated enhancement of PMX 50003 against E. coli ATCC 25922 via TEM. Panel A: control; Panel B: PMX 50003 (1× MIC), $15 \mathrm{~min}$; Panel C: PMX 50003 (1× MIC) + $200 \mu \mathrm{M}$ nerolidol, $5 \mathrm{~min}$. Although PMX 50003 alone at $15 \mathrm{~min}$ exposure caused some damage to cells, the combination of nerolidol and PMX 50003 led to severe damage (blebbing) after only 5 min. These data provide evidence of cooperative interactions between nerolidol and this biomimetic polymer. 


\begin{tabular}{|c|c|c|}
\hline Property/Attribute & Natural AMPs & Synthetic AMP Mimics \\
\hline Cost to produce & Expensive - large-scale synthesis not cost-effective & $\begin{array}{l}\text { Less expensive - some mimics produced via "one pot" } \\
\text { synthesis }\end{array}$ \\
\hline $\begin{array}{l}\text { Stability (in vivo, shelf } \\
\text { stability) }\end{array}$ & $\begin{array}{l}\text { Susceptible to proteases; sequence-dependent shelf stability } \\
\text { (may be susceptible to chemical hydrolysis, oxidation, } \\
\text { deamidation, etc.) }\end{array}$ & $\begin{array}{l}\text { Not a substrate for proteases; small compounds or } \\
\text { polymers may be intrinsically robust and stable }\end{array}$ \\
\hline Structural malleability & Not readily modifiable & $\begin{array}{l}\text { 1) Parent molecule can be modified with different side } \\
\text { groups during synthesis } \\
\text { 2) Rational design of parent molecules and introduction } \\
\text { of modifications supported by computer-assisted } \\
\text { modeling and design }\end{array}$ \\
\hline Antimicrobial Spectrum & Variable; intrinsic property of AMP & $\begin{array}{l}\text { Mimics may be designed and screened for specific spectra } \\
\text { or activities ("fine tuning") }\end{array}$ \\
\hline Antigenicity & $\begin{array}{l}\text { AMPs proteinaceous - may be recognized by body as a } \\
\text { foreign protein (problematic for systemic application) }\end{array}$ & $\begin{array}{l}\text { Mimics entirely synthetic, may be developed to have } \\
\text { reduced (or no) antigenicity }\end{array}$ \\
\hline $\begin{array}{l}\text { Salt and divalent cation } \\
\text { effects }\end{array}$ & Decreased potency in presence of salt/cations problematic & $\begin{array}{l}\text { Mimics may be designed and screened for activity under } \\
\text { physiologically relevant conditions }\end{array}$ \\
\hline $\begin{array}{l}\text { Off-target effects (hemolysis, } \\
\text { cytotoxicity) }\end{array}$ & Variable; if present, are intrinsic property of AMP & $\begin{array}{l}\text { Mimics may be designed and screened to minimize off- } \\
\text { target effects }\end{array}$ \\
\hline
\end{tabular}

This study demonstrates antimicrobial activities of biomimetic polymers against a broad range of microorganisms. In addition, the enhancement effects of sesquiterpenoids, previously proven effective in combination with clinically important antibiotics (Brehm-Stecher \& Johnson, 2003), have been extended to include these polymers, leading to the production of dramatically effective binary antimicrobial formulation. All three polymers exhibited antimicrobial activity against both gram-positive and gram-negative bacteria as well as against fungi and molds, with substantial increases in activity demonstrated in the presence of the three different sesquiterpenoids. Additional experiments determined that the combination of polymers and sesquiterpenoids significantly decreased initial contact time necessary for the inhibition of growth when compared to controls. Further analysis of the mechanisms of action showed that penetration of the outer membrane was increased in the presence of the sesquiterpenoids, pointing to increased permeability as a mechanism for the increased activity of the polymers when used in combination. These combinations have the potential to yield high-activity antimicrobial systems that have excellent promise for addressing our dire need for methods to combat life-threatening bacterial infections caused by organisms resistant to traditional antimicrobials. Because our approach targets a generic and highly conserved bacterial structure - the cell membrane - it is unlikely that targeted microorganisms will be able to develop additional resistance to this approach. The formulations developed here may ultimately be of use both in clinical and therapeutic applications and potentially in food-related applications, such as improved, wide-spectrum sanitizers.

\section{Acknowledgements}

The authors thank PolyMedix, Inc. (Radnor, PA) for supplying the biomimetic polymers used in this study and Jean Ross at the University of Iowa's Central Microscopy Research Facility (CMRF, Iowa City, IA) for her expert electron microscopy work. Funding for this work was provided by PolyMedix, Inc., Iowa State University's Office of Biotechnology and by USDA National Needs Fellowship Grant No. 2005-02324.

\section{References}

Branen, J. K., \& Davidson, P. M. (2004). Enhancement of nisin, lysozyme, and monolaurin antimicrobial activities by ethylenediaminetetraacetic acid and lactoferrin. International Journal of Food Microbiology, 90, 63-74. http://dx.doi.org/10.1016/S0168-1605 (03)00172-7.

Brehm-Stecher, B. F., \& Johnson, E. A. (2003). Sensitization of Staphylococcus aureus and Escherichia coli to antibiotics by the sesquiterpenoids nerolidol, farnesol, bisabolol, and apritone. Antimicrobial Agents and Chemotherapy, 47(10), 3357-3360. http://dx.doi.org/10.1128/AAC.47.10.3357-3360.2003.

CLSI. (2006). Performance Standards for Antimicrobial Disk Susceptibility Tests; Approved Standard-Ninth Edition. CLSI document M2-A9. Wayne, PA: Clinical and Laboratory Standards Institute.

CLSI. (2008a). Reference method for broth dilution antifungal susceptibility testing of filamentous fungi; Approved standard Second Edition. CLSI document M38-A2. Wayne, PA: Clinical and Laboratory Standards Institute.

CLSI. (2008b). Reference method for broth dilution antifungal susceptibility testing of yeasts; Approved Standard - Third Edition. CLSI document M27-A3. Wayne, PA: Clinical and Laboratory Standards Institute.

CLSI. (2012). Methods for Dilution Antimicrobial Susceptibility Tests for Bacteria That Grow Aerobically; Approved Standard-Ninth Edition. CLSI document M07-A9. Wayne, PA: Clinical and Laboratory Standards Institute.

Difco \& BBL Manual: Manual of Microbiological Culture Media. (2009). M. J. Zimbro \& D. A. Power (Eds.), "D/E Neutralizing Agar $\bullet D / E$ Neutralizing Broth" (2nd ed., p. 172). Sparks, MD: Becton, Dickinson and Company.

Espinel-Ingroff, A., \& Canton, E. (2007). Antifungal susceptibility testing of filamentous fungi. In R. Schwalbe, L. Steele-Moore, \& A. C. Goodwin (Eds.), Antimicrobial Susceptibility Testing Protocols (pp. 209-241). Boca Raton, FL: CRC Press. 
Hancock, R. E. W. (2001). Cationic peptides: Effectors in innate immunity and novel antimicrobials. Lancet, 1, 156-164. http:// dx.doi.org/10.1016/S1473-3099(01)00092-5.

Hancock, R. E. W., \& Rozek, A. (2002). Role of membranes in the activities of antimicrobial cationic peptides. FEMS Microbiology Letters, 206, 143-149. http://dx.doi.org/10.1111/j.1574-6968. 2002.tb11000.x.

Inoue, Y., Shiraishi, A., Hada, T., Hirose, K., Hamashima, H., \& Shimada, J. (2004). The antibacterial effects of terpene alcohols on Staphylococcus aureus and their mode of action. FEMS Microbiology Letters, 237, 325-331. http://dx.doi.org/10.1111/j.15746968.2004.tb09714.x.

Jay, J. (2005). Food Protection with High Temperatures, and Characteristics of Thermophilic Microorganisms. In J. M. Jay, M. J. Loessner, \& D. A. Golden (Ed.), Modern Food Microbiology, (7th ed., pp. 423). New York, NY: Springer.

Jett, B. D., Hatter, K. L., Huycke, M. M., \& Gilmore, M. S. (1997). Simplified agar plate method for quantifying viable bacteria. BioTechniques, 23, 648-650.

Kuroda, K., \& DeGrado, W.F. (2005). Amphiphilic polymethacrylate derivatives as antimicrobial agents. Journal of the American Chemical Society, 127, 4128-4129. http://dx.doi.org/10.1021/ ja044205+.

Lambert, R. J., Johnston, M. D., \& Simons, E.-A. (1998). Disinfectant testing: Use of the Bioscreen Microbiological Growth Analyser for laboratory biocide screening. Letters in Applied Microbiology, 26, 288-292. http://dx.doi.org/10.1046/j.1472-765X.1998.00334.x.

Lockwood, N. A., \& Mayo, K. H. (2003). The future for antibiotics: Bacterial membrane disintegrators. Drugs Future, 28(9), 911-923. http://dx.doi.org/10.1358/dof.2003.028.09.857379.

Santos, D. A., Barros, M. E. S., \& Hamdan, J. S. (2006). Establishing a method of inoculum preparation for susceptibility testing of Trichophyton rubrum and Trichophyton mentagrophytes. Journal of Clinical Microbiology, 44(1), 98-101. http://dx.doi.org/10. 1128/JCM.44.1.98-101.2006.

Shai, Y. (2002). Mode of action of membrane active antimicrobial peptides. Biopolymers, 66, 236-248. http://dx.doi.org/10.1002/ bip. 10260 .
Tew, G. N., Liu, D., Chen, B., Doerksen, R. J., Kaplan, J., Carroll, R. J., et al. (2002). De novo design of biomimetic antimicrobial polymer. Proceedings of the National Academy of Sciences of the United States of America, 99(8), 5111-5114. http://dx.doi.org/10.1073/ pnas.082046199.

Vaara, M. (1992). Agents that increase the permeability of the outer membrane. Microbiology Reviews, 56(3), 395-411.

Yeaman, M. R., \& Yount, N. Y. (2003). Mechanisms of antimicrobial peptide action and resistance. Pharmacological Reviews, 55, 27-55. http://dx.doi.org/10.1124/pr.55.1.2.

Zasloff, M. (2002). Antimicrobial peptides of multicellular organisms. Nature, 415, 389-395. http://dx.doi.org/10.1038/ 415389a.

\section{COMPETING INTERESTS}

The authors declare no competing interests.

\section{PUBLISHING NOTES}

(C) 2017 Wright and Brehm-Stecher. This work has been published open access under Creative Commons Attribution License CC BY 4.0, which permits unrestricted use, distribution, and reproduction in any medium, provided the original work is properly cited. Conditions, terms of use and publishing policy can be found at www.scienceopen.com.

Please note that this article may not have been peer reviewed yet and is under continuous post-publication peer review. For the current reviewing status please click here or scan the QR code on the right.

\section{ScienceOPEN.com}

\section{research+publishing network}

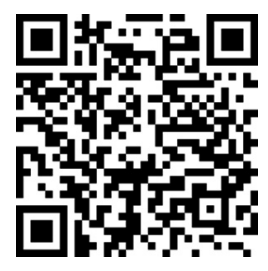

